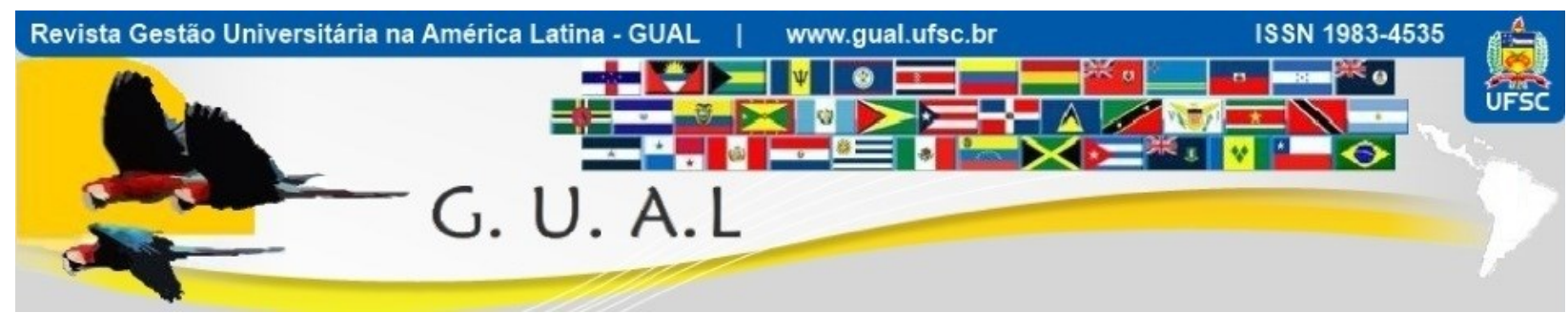

DOI: http://dx.doi.org/10.5007/1983-4535.2014v7n1p148

\title{
ORÇAMENTO POR DESEMPENHO: UMA ANÁLISE DA RELAÇÃO ENTRE DESEMPENHO E ALOCAÇÃO DE RECURSOS EM AÇÕES NO ORÇAMENTO DE UMA INSTITUIÇÃO FEDERAL DE ENSINO SUPERIOR
}

\section{PERFORMANCE-BASED BUDGETING: AN ANALYSIS OF THE RELATIONSHIP BETWEEN PERFORMANCE AND RESOURCES ALLOCATION IN A FEDERAL INSTITUTION OF HIGHER EDUCATION IN BRAZIL}

Maurício Vasconcellos Leão Lyrio, Doutorando Universidade Federal de Santa Catarina - UFSC mauriciovll@gmail.com

Verônica de Souza Melo, Mestranda Universidade Federal de Santa Catarina - UFSC vesouza@hotmail.com

Maurício Mello Codesso, Mestrando Universidade Federal de Santa Catarina - UFSC mmcodesso@gmail.com

Rogério João Lunkes, Doutor Universidade Federal de Santa Catarina - UFSC lunkes@cse.ufsc.br

Recebido em 13/março/2013

Aprovado em 27/novembro/2013

Sistema de Avaliação: Double Blind Review

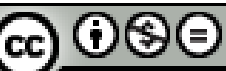

Esta obra está sob uma Licença Creative Commons Atribuição-Uso. 


\title{
RESUMO
}

O presente artigo se insere no contexto da discussão sobre a alocação de recursos públicos, especificamente no âmbito de uma Instituição Federal de Ensino Superior - IFES - que nesse caso se configura como objeto de estudo. A partir de uma inquietação por parte dos autores em relação à utilização das informações sobre o desempenho no planejamento orçamentário da Universidade Federal de Santa Catarina - UFSC busca responder à seguinte pergunta de pesquisa: Existe correlação positiva entre o desempenho alcançado nos indicadores e os recursos alocados às ações orçamentárias no âmbito da Universidade Federal de Santa Catarina? Para o alcance de seu objetivo, os autores seguem uma corrente de pesquisa que surge de forma alinhada ao movimento por resultados iniciado com a crise fiscal dos anos de 1980, a saber, Performance-based Budgeting - PBB - ou orçamento por resultados ou desempenho. No que tange à metodologia o estudo se configura como descritivo, adotando uma abordagem quantitativa e utilizando-se de estatística descritiva para análise dos dados. Os resultados demonstraram fraca correlação entre o desempenho das ações do orçamento da instituição analisada e os recursos alocados a essas ações.

Palavras-Chaves: Setor Público. Orçamento. Orçamento por Desempenho. Instituição Pública de Ensino superior.

\begin{abstract}
This article is located in the discussion of allocation of public resources, specifically in the context of a Federal Higher Education Institution - IFES - which in this case is the object of study. From a discomfort on the part of the authors regarding the use of performance information in budgeting of the Federal University of Santa Catarina - UFSC it aims to answer the following research question: Is there a positive correlation between the achieved performance indicators and the resources allocated to budget actions under the Federal University of Santa Catarina? To achieve its goal, the authors follow a line of research that appears in alignment with the so called movement for results which began with the fiscal crisis of the 1980s, namely, Performance-based Budgeting - PBB - or budget for results or performance. With regard to the study methodology it is framed as descriptive, adopting a quantitative approach and using descriptive statistics to analyze the data. The results demonstrated a weak correlation between the performance of the actions of the analyzed institution's budget and the resources allocated to these actions.
\end{abstract}

Key Words: Public Sector; Budgeting; Performance-based Budgeting; Public Institution of Higher Education. 


\section{INTRODUÇÃO}

Nas ultimas três décadas a administração pública vem incorporando em seu escopo as mudanças do mundo contemporâneo e a evolução do pensamento administrativo praticado na iniciativa privada. Estas mudanças buscam, entre outras questões, melhorar a alocação de recursos de maneira eficiente, de modo a proporcionar benefícios à sociedade, transparência em suas operações e o alcance dos objetivos propostos em suas diversas esferas, seja municipal, estadual ou federal.

A discussão sobre uma melhor alocação de recursos para atendimento das necessidades da sociedade é tema recorrente em pesquisas, bem como iniciativas para melhoria da alocação de recursos tem sido desenvolvidas e implementadas. Dentre essas iniciativas destacam-se aquelas ligadas aos sistemas de planejamento e controle, sendo que o orçamento tem recebido significativa atenção na literatura. Além disso, estudos empíricos mostram que o orçamento é amplamente utilizado pelas organizações (LEITE et al., 2008; LIBBY; LINDSAY, 2010; OSTERGREN; STENSAKER, 2011; UYAR, 2009).

O presente artigo se insere no contexto da discussão sobre a alocação de recursos públicos, especificamente no âmbito de uma Instituição Federal de Ensino Superior - IFES que nesse caso se configura como objeto de estudo. O trabalho está alinhado aos estudos desenvolvidos no âmbito da $2^{\mathrm{a}}$ reforma da administração pública, denominada New Public Management - NPM, considerado um "[...] modelo normativo pós-burocrático para a estruturação e a gestão da administração pública baseado em valores de eficiência, eficácia e competitividade" (SECCHI, 2009, p. 354). O estudo surge a partir de uma inquietação por parte dos pesquisadores envolvidos, em relação à utilização das informações sobre o desempenho no planejamento orçamentário da Universidade Federal de Santa Catarina UFSC e busca responder à seguinte pergunta de pesquisa: Existe correlação positiva entre o desempenho alcançado nos indicadores e os recursos alocados às ações orçamentárias no âmbito da Universidade Federal de Santa Catarina?

Frente a este contexto de transformação no qual as organizações públicas estão inseridas, faz-se importante entender se os recursos aplicados na instituição em estudo Universidade Federal de Santa Catarina - estão efetivamente colaborando para a melhoria de seu desempenho. De acordo com Waggoner (1999), o uso de indicadores busca evidenciar o desempenho de determinada atividade ou processo gerencial. Para o autor, esse desempenho é 
influenciado por relações de poder e coalizões de interesses, bem como por questões de legislação e aspectos relacionados à implementação e gestão de processos políticos. Especificamente no caso das Instituições Federais de Ensino Superior - IFES, a relevância da questão se dá pelo seu objetivo, a saber, a promoção do progresso intelectual da população. Esse tipo de instituição tem seu orçamento submetido pelo Ministério da Educação e Cultura - MEC - e aprovado pela União para que possam desenvolver suas atividades e implementar seu planejamento, sendo seus recursos alocados em ensino, pesquisa e extensão, assim como em manutenção do espaço físico para o desenvolvimento de suas atividades, o que vem a justificar o desenvolvimento de pesquisas sobre o tema.

Para o alcance do objetivo da pesquisa, os autores seguem uma corrente de pesquisa que surge de forma alinhada ao movimento por resultados iniciado com a crise fiscal dos anos de 1980, denominada Performance-based Budgeting - PBB - ou orçamento por resultados ou desempenho. Considerada uma estratégia operacional desenvolvida no âmbito da NPM, o orçamento por desempenho busca aprimorar o processo de elaboração, execução e controle, priorizando os resultados em detrimento dos mecanismos de comando e controle (HO, 2011). As pesquisas desenvolvidas no âmbito do PBB visam analisar a integração entre as informações de desempenho e os processos de planejamento e orçamento, sendo esta também a contribuição a ser aqui apresentada, em estudo desenvolvido no âmbito de uma IFES.

Após essa seção introdutória o estudo se organiza da seguinte forma: a seção 2 referencial teórico - se propõe a tecer considerações sobre orçamento e orçamento público; apresentar a base teórica utilizada no estudo, a saber, o performance-based budgeting - PBB; bem como apresentar outros estudos sobre orçamento em Instituições de Ensino Superior. Em seguida - seção 3 - será apresentado o enquadramento metodológico da pesquisa e os procedimentos adotados para seleção do referencial teórico e análise dos dados obtidos em campo. A seção 4 apresenta a IFES utilizada como objeto de estudo e os resultados da análise. Finalmente a seção 5 se propõe a tecer as considerações finais a respeito do estudo realizado.

\section{REFERENCIAL TEÓRICO}

O referencial teórico do presente estudo se divide em três eixos, a saber: (i) considerações sobre orçamento e orçamento público no Brasil - com vistas a situar o leitor em relação ao tema do estudo; (ii) performance-based budgeting: evolução histórica e conceitos principais - com vistas a apresentar a base teórica utilizada para análise dos dados; e, (iii) 
Orçamento em Instituições Públicas de Ensino Superior: pesquisas anteriores - com vistas a apresentar outros estudos abordando a temática orçamentária no âmbito das Instituições Públicas de Ensino Superior brasileiras.

\subsection{CONSIDERAÇÕES SOBRE ORÇAMENTO E ORÇAMENTO PÚBLICO NO BRASIL}

O termo orçamento pode ser definido como um plano abrangente, considerando todas as fases das operações de um dado negócio, com foco em um período futuro definido. Lunkes (2009, p. 27) argumenta que o orçamento é "[...] um plano de ação futuro da administração para determinado período", abrangendo tanto aspectos financeiros quanto aspectos não financeiros, e funcionando como direcionamento a ser seguido por uma organização em períodos vindouros. Como uma atividade ligada ao planejamento, execução e controle nas organizações, o orçamento apresenta uma série de vantagens, entre elas, a exigência de definição prévia de objetivos, diretrizes e políticas de desempenho; a indução da comunicação, integração e participação ao longo de toda a organização; foco no futuro por parte dos colaboradores; formação de uma estrutura com atribuições de responsabilidade; promoção de visão sistêmica; aumento da coordenação e controle das atividades entre outras (LUNKES, 2009)

Por outro lado, a utilização de práticas orçamentárias também possui algumas desvantagens, como a inflexibilidade, o longo tempo destinado a sua elaboração, o condicionamento às forças de poder em uma organização, reações indesejadas, visão apenas financeira e desmotivação. Boisvert (1999) complementa essa questão chamando a atenção para questões como o uso excessivo de tendências históricas para estabelecimento de objetivos; a aplicação de percentuais de cortes gerais nos custos, sem análise prévia do contexto de cada área; a análise defasada no tempo, impossibilitando a correção imediata de anomalias; a excessiva associação dos custos na preparação do orçamento; bem como a incapacidade de adaptação a mudanças no ambiente da organização.

No caso do Brasil, diversas atualizações e alterações em relação a legislação orçamentária tem tido lugar, culminando nas novidades oriundas da Constituição de 1988, que deu competência ao Poder Legislativo em propor emendas ao Projeto de Lei do Orçamento, bem como passou a exigir do Poder Executivo o encaminhamento anual do projeto de Lei das Diretrizes Orçamentárias, com vistas a orientar a elaboração da Lei Orçamentária e dispondo sobre a política de fomento a ser observada pelas agências de governo (GIACOMONI, 2010) 
A Constituição de 1988 instituiu 3 instrumentos para planejamento e implementação de políticas públicas, a saber, a Lei do Plano Plurianual (PPA), a Lei das Diretrizes Orçamentárias (LDO) e a Lei do Orçamento Anual (LOA). O PPA se constitui em uma síntese do planejamento de toda a administração pública, e serve de orientação para a elaboração dos demais planos e programas de governo de forma regionalizada, envolvendo as diretrizes, objetivos e metas para despesas de capital, as despesas decorrentes das despesas de capital e os programas de duração continuada. A LDO, por sua vez, compreende as metas e prioridades da administração pública para o exercício subsequente, bem como orienta a elaboração da LOA, que envolve 3 orçamentos, o físcal, o da seguridade social e o de investimento das empresas. Além disso, a Lei de Responsabilidade Fiscal (LRF) ampliou a importância da LDO, atribuindo-lhe novas prerrogativas, dentre elas, a incumbência de disciplinar temas específicos ligados ao equilíbrio entre receitas e despesas públicas, metas e riscos fiscais, programação financeira e cronograma de execução mensal.

Atualmente essa é a configuração da estrutura para planejamento orçamentário no Brasil. Apesar de possuir sua sistemática estabelecida em legislação, conforme citado por Cavalcante (2010), os orçamentos públicos convivem com um fator limitador das escolhas alocativas, como a sua rigidez. Além disso, quanto maior o tamanho e a abrangência do orçamento, mais ele se torna inflexível e restrito. No caso do setor público, é evidente "[...] a prática cotidiana do orçamento incrementalista, que consiste na tomada de decisões fundamentadas em ajustes marginais, baseados nas dotações dos exercícios anteriores" (CAVALCANTE, 2010, p.15).

Autores como Larkley e Devereux (1999), Radin (2006), Robinson e Marc (2002), Rubin (2010) argumentam que se o processo orçamentário é direcionado prioritariamente por jogos de poder, valores e considerações políticas, este se torna uma arena na qual existe uma competição entre os resultados almejados pela sociedade e os interesses políticos, sendo esta a problemática na qual o estudo se insere.

\subsection{PERFORMANCE-BASE BUDGENTING: EVOLUÇÃO HISTÓRICA E CONCEITOS PRINCIPAIS}

O Performance-Based Budgeting - PBB - ou orçamento por desempenho é

[...] considerado uma estratégia operacional desenvolvida no âmbito do New Public

Management (NPM), ou Nova Gestão Pública. O movimento por resultados nas 
ações do governo originou-se da situação de crise fiscal que assolou diversos países a partir da década de 80 e das pressões da sociedade civil por melhorias na eficiência e efetividade dos serviços públicos. (CAVALCANTE, 2010, p. 15)

Sua origem remonta ao modelo Planning, Programming e Budgenting System (PPBS), desenvolvido nos Estados Unidos na década de 1960. A partir desse modelo inicial, foram desenvolvidas inovações no processo orçamentário que culminou no surgimento do PBB, no início da década de 1990 (CAVALCANTE, 2010). Tat-Kei Ho (2011) salienta que pesquisas do tipo survey, realizadas nos municípios e estados norte-americanos, já demonstravam que a integração entre medidas de desempenho e processos orçamentários se tornou popular a partir dos anos de 1980. Sendo que o PBB agrega valor ao diálogo sobre orçamento durante diferentes fases do processo orçamentário, se tornando um instrumento útil para apoiar a tomada de decisão nesse contexto.

Kong (2005) ao tratar do tema, argumenta que a avaliação de desempenho como um mecanismo integrado para planejamento, gestão e orçamentação é ideia antiga. Essa abordagem já fazia parte da administração científica na virada do séc. XIX e o PBB pode ser entendido como uma reformulação dessas práticas.

A chamada primeira onda do PBB nos Estados Unidos se deu no início da década de 1940, por meio de publicação sobre a mensuração das atividades municipais produzida pelo International City/County Management Association (ICMA) e em nível federal, por meio das recomendações da comissão Hoover em 1949, se mantendo como uma filosofia de gestão através dos tempos. Com o surgimento de outras reformas orçamentárias nos E.U.A., como o Planning-Program-Budgeting (PPB) e o Zero-Based Budgeting (ZBB) o uso do PBB declinou nas décadas de 1960 e 1970. Na prática, uma visão pessimista ligada ao entendimento de que os custos de implementação de sistemas dessa complexidade superava seus benefícios acabou prevalencendo (KONG, 2005).

Apesar da discussão a respeito da complexidade de implementação desse tipo de sistema orçamentário, pesquisas continuaram a ser desenvolvidas com diferentes focos. Já no início da década de 1990, reforçado pelo movimento de reinvenção do governo proposto por Osborne e Glaeber e aliado a iniciativa do National Performance Review do vice-presidente Al Gore, o PBB ganhou uma nova vida. Nessa chamada segunda onda,

[...] PBB was launched with political support and more importantly was fueled by some practical advantages. First, the Second Wave is better situated than the First Wave with more details of performance measurement. Second, increased use of 
computer technology and management applications have made it possible to process more data in a timely and cost-saving manner. Third, performance measures have been advocated not only as part of budgeting system but also as an integral element of strategic planning and management (KONG, 2005, p.94).

A grande mudança implementada nessa segunda onda do PBB foi a alteração da lógica de "usar ou perder" o orçamento para uma nova lógica, de criar uma estrutura adequada e incentivos baseados em resultados. Entretanto, desenvolver a capacidade institucional de utilizar as informações de desempenho leva tempo, em pesquisa realizada por Grizzle e Pettijohn (2002), é relatado que, apesar do Estado da Pennsylvania ter implementado o PBB em 1970, somente em 1975 os dados sobre o desempenho do governo foram utilizados para elaboração do orçamento. No decorrer desse período foi necessário desenvolver o entendimento por parte dos executivos e do pessoal de staff do governo a respeito dessa abordagem.

Para Cavalcante (2010), o PBB objetiva modificar a elaboração orçamentária, dando ênfase aos resultados ao invés de considerar apenas os mecanismos de controle. Nesse sentido, argumenta que as informações da avaliação dos programas governamentais servem de subsídio para a tomada de decisão alocativa, bem como para o planejamento estratégico e accountability dos gestores responsáveis pelo orçamento. Para Brumby e Robinson (2005), o PBB é um modelo orçamentário que busca relacionar, por um lado, os recursos alocados aos programas de governo e, por outro, os resultados mensuráveis das políticas públicas, desta forma, permite a geração de informações que auxiliam o processo de tomada de decisão pelos governantes e gestores públicos.

\subsection{ORÇAMENTO EM INSTITUIÇÕES PÚBLICAS DE ENSINO SUPERIOR: PESQUISAS ANTERIORES}

A temática do orçamento em instituições públicas de ensino superior está inserida no contexto geral das pesquisas acerca de orçamento público, a partir do qual é possível realizar um recorte mais específico. Nesse sentido, Brotti, Laffin e Borgert (2007) afirmam que pesquisas sobre o tema orçamento, distribuição orçamentária ou processo orçamentário, no período de 1980 a 2005 na área de contabilidade aparecem em número relevante e se configuram como um campo de pesquisa a ser explorado.

Calil (2000) abordou o processo de elaboração de propostas orçamentárias na Universidade Estadual do Paraná, com o propósito específico de conhecer o rito deste 
processo, sob a ótica dos técnicos da instituição. Como resultado, a pesquisa apontou a necessidade de uma capacitação que instrumentalizasse os técnicos que lidavam com o orçamento da instituição nos seus diversos níveis em assuntos como contabilidade pública e planejamento. Segundo a autora, a instituição de ensino afirma que, quando há integração com a comunidade acadêmica nos processos de execução e avaliação, o processo orçamentário torna-se um instrumento democrático.

Pires (2001) aborda a questão da autonomia das universidades públicas através do estudo de caso da Universidade Estadual de Maringá. Nessa pesquisa, o autor faz uma revisão geral do processo orçamentário, descrevendo as várias etapas do ciclo orçamentário, a lei de responsabilidade fiscal e as premissas orçamentárias para a autonomia financeira, além de diferenciar a autonomia universitária em três diferentes abordagens: A autonomia didáticocientífica, administrativa e de gestão financeira e patrimonial, esta última, tema central do trabalho. Dentre os resultados da pesquisa pode-se destacar o levantamento de fatores que dificultam e facilitam a plenitude da autonomia financeira da Universidade Estadual de Maringá.

Calil (2005) também evidencia a questão da autonomia das universidades na gestão de seus recursos orçamentários, mas com um foco na integração orçamentária, financeira e de custos nas universidades estaduais do Paraná. Deste modo a autora buscou investigar nas instituições públicas de ensino superior estaduais, como se relacionavam as questões de custeio por aluno e curso, a representatividade dos custos alocados no orçamento, e como se poderia realizar uma integração orçamentária, financeira e de custos. Para tanto Calil (2005) propõe um Sistema de Integração Orçamentária e de Custos (SIOFIC), baseado nas exigências da legislação brasileira, o qual foi validado em sua tese, demonstrando indícios de viabilidade para aplicação nas instituições de ensino e demais órgãos e entidades estaduais.

Pires (2005) segue com a preocupação na eficiência dos gastos públicos nas universidades públicas do estado do Paraná ao sugerir uma proposta inovadora de gestão orçamentária: Orçamento Baseado em Desempenho Acadêmico (OBDA). O modelo proposto é estruturado em três módulos diferenciados (Resolução de Diretrizes Orçamentárias - RDO; Método de Cálculo para Alocação Interna de Recursos Orçamentários - MC/ODC e Demonstrativo de Receitas e Despesas - DRD) que, quando integrados formam um modelo de orçamento fundamentado na alocação de recursos orçamentários entre as unidades internas com base na produção acadêmica. 
Paiva (2009) traz o debate do orçamento público para o campo das políticas públicas e gestão da educação, estudando as políticas de financiamento para a educação superior através da avaliação institucional e gestão orçamentária na Universidade Federal de Uberlândia. A autora procurou relacionar as políticas públicas de educação aos investimentos realizados na universidade e suas fontes de financiamento (públicas e privadas), através da gestão orçamentária da entidade.

Vasconcelos (2010) aborda a temática do orçamento público nas instituições federais de ensino superior, com o caso da Universidade Federal de Sergipe, no qual descreve as peculiaridades do processo orçamentário na universidade, as potencialidades e perspectivas para o aumento de receita, a alocação das despesas e investimentos e, por fim, analisa o desempenho da universidade de 1998 a 2008, buscando retratar o orçamento como um espelho do planejamento para o período. Tal integração entre planejamento e orçamento público também foi abordada por Francisco (2005). O autor realizou a pesquisa com maior abrangência através de um estudo multicaso em 55 Instituições Federais de Ensino Superior (IFES), colhendo opinião dos responsáveis pelo planejamento nas respectivas instituições. O resultado da pesquisa apontou que os entrevistados reconhecem o orçamento público como um importante instrumento de planejamento, mas que ele pode não representar as reais necessidades da instituição, precisando ser complementado com um plano de desenvolvimento institucional.

Outras temáticas acerca de orçamento público também podem ser percebidas nas pesquisas sobre o tema realizadas no portal da CAPES, como as políticas públicas de financiamento do ensino Público Superior Brasileiro, abordada por Araújo (2009) e Paiva (2009), e Orçamento Participativo nas Universidades, por Ferreira (2003). O presente artigo dá prosseguimento a este tipo de estudo, visando analisar a relação existente entre o desempenho dos indicadores e os recursos alocados nas ações do orçamento no âmbito da Universidade Federal de Santa Catarina - UFSC.

\section{METODOLOGIA}

A metodologia utilizada para realização do presente estudo se apresenta divida em três eixos, a saber: (i) o enquadramento metodológico; (ii) o processo para construção do referencial teórico; e, (iii) a apresentação do método estatístico adotado para análise dos dados. 
No que tange ao enquadramento metodológico, este estudo assume caráter descritivo uma vez que busca analisar a relação existente entre desempenho e alocação de recursos no âmbito da UFSC. Para o tratamento dos dados adota uma abordagem quantitativa, uma vez que serão tabulados e tratados por meio de estatística descritiva e de análise de correlação. Os dados serão levantados pelos pesquisadores e tratados em planilhas Excel ${ }^{\circledR}$, sendo que existe preocupação em descrever a forma de levantamento desses dados, com vistas a permitir sua replicabilidade e análise temporal, o que se configura como uma das preocupações em relação a pesquisas quantitativas, conforme descrito por Bryman (1988a, 1988b). Finalmente, quanto aos procedimentos técnicos, constitui-se em um trabalho documental (GIL, 2007; RICHARDSON, 1999), nesse caso materializado nos artigos selecionados para o estudo e nos dados que emergirem dos documentos internos analisados.

Em relação aos procedimentos para seleção do referencial teórico, os estudos anteriores sobre orçamento em instituições de ensino foram selecionados por meio de busca no portal de periódicos da CAPES, utilizando como palavras-chave os termos "orçamento", "orçamento público", "orçamento nas universidades" e "orçamento nas instituições de ensino superior", a partir dos resultados encontrados foram selecionados os trabalhos com maior aderência ao tema do presente artigo. No que tange a seleção dos artigos que abordam o tema orçamento por desempenho, foi também utilizada a base de dados da CAPES por meio de um levantamento estruturado e não intencional, realizado em 4 etapas, conforme proposto por Rosa et. al. (2011). Inicialmente foi utilizada como palavra-chave para identificação da população da pesquisa o termo "performance based budgeting"; em seguida foi realizado refinamento dos resultados restringindo os resultados ao nível superior "periódicos revisados por pares"; tópico "public administration”; data de publicação "2002 até 2006 e após 2006", com vistas a trabalhar com os artigos desenvolvidos na última década; e, idioma "inglês". Os artigos resultantes do refinamento da busca tiveram seus resumos lidos para verificação da aderência dos mesmos ao tema da pesquisa. $\mathrm{O}$ resultado final desse tratamento de dados se configura como a amostra de artigos utilizada no referencial teórico.

Finalmente, com relação ao método estatístico adotado no tratamento dos dados, com vistas a testar empiricamente a relação existente entre o desempenho das ações no orçamento da UFSC com base nos indicadores associados e os recursos alocados a essas ações, foi utilizado como instrumento de intervenção a análise de correlação, inspirado na proposta de Tat-Kei Ho (2011). Foram utilizadas para análise duas variáveis de controle, o 
valor do orçamento e o valor do indicador de desempenho associado a cada ação do orçamento.

Os dados utilizados na pesquisa foram extraídos dos relatórios de gestão da UFSC, referentes ao período de 2007 a 2011, e posteriormente transformados em um indicador de performance, tornando possível a comparação entre os diversos valores de orçamento e diferentes indicadores físicos de cada programa e ação do orçamento. O Quadro 1 apresenta o indicador de performance utilizado no estudo

\section{Indicador de Performance}

$\Delta$ execução orçamentária / $\Delta$ desempenho físico da ação

Quadro 1: Indicador de Performance.

Fonte: Elaborado pelos Autores.

Para a análise realizada foram estabelecidas duas hipóteses, a saber: $\mathrm{Hl}=\mathrm{O}$ desempenho da ação em determinado ano influencia o orçamento do ano posterior; e, H2 = o aumento do orçamento em relação ao orçamento do ano anterior influencia o desempenho da ação no ano seguinte. No caso da H1, a confirmação da hipótese evidencia a utilização das informações de desempenho no momento de alocação de recursos, tendo em vista que as ações que obtiverem melhor desempenho tendem a receber mais recursos no ano seguinte. A H2 busca verificar se, nos casos em que houve baixo desempenho na ação e, mesmo assim, houve aumento do orçamento no ano seguinte, a injeção de mais recursos tende a melhorar o desempenho da ação no ano seguinte. Passa-se a seguir à apresentação e análise dos resultados da pesquisa.

\section{APRESENTAÇÃO E ANÁLISE DOS RESULTADOS}

A presente seção se propõe a apresentar os resultados do estudo. Nesse sentido, a seção 4.1 busca apresentar a instituição utilizada como objeto de pesquisa, enquanto a seção 4.2 apresenta a aplicação do método estatístico proposto e tece uma análise crítica a partir dos resultados apresentados.

\subsection{APRESENTAÇÃO DA ORGANIZAÇÃO: A UNIVERSIDADE FEDERAL DE SANTA CATARINA}

Criada em dezembro de 1960 a Universidade Federal de Santa Catarina (UFSC) conta atualmente com um patrimônio de cerca de 20 milhões de metros quadrados distribuídos no 
estado de Santa Catarina, especialmente nos municípios de Florianópolis, Araranguá, Joinville e Curitibanos, onde possui campi, e nos municípios de Araquari e Camboriú onde tem instalado colégios agrícolas. Em 2011, a Universidade possuía 29.177 alunos matriculados na graduação e 13.867 pós-graduandos, além de 1.167 alunos na educação básica. Considerando o universo de servidores técnicos e docentes, a UFSC pode ser comparada a um município de cerca de 45 mil moradores. Para gerir tamanha estrutura, entre os anos de 2007 a 2011, segundo informações apresentadas em relatório disponibilizado na internet pela Diretoria de Gestão Orçamentária da UFSC, a Universidade teve em média cerca de 790 milhões de reais de orçamento anual.

A Universidade é uma autarquia da administração indireta e como Unidade Orçamentária vinculada ao Ministério da Educação e Cultura (MEC) recebe dotações próprias, as quais são consignadas via orçamento da União. Enquanto no plano nacional a estrutura hierárquica do orçamento compõe-se da elaboração do Plano Plurianual (PPA), da Lei de Diretrizes Orçamentárias (LDO) e da Lei Orçamentária Anual (LOA); a Universidade possui uma dinâmica interna própria de planejamento e orçamento que se integra ao plano nacional quando da elaboração do orçamento anual.

UFSC não precisa apresentar o PPA, tendo em vista que se configura como uma unidade orçamentária do órgão MEC, conforme a classificação estabelecida nos artigos 13 e 14 da Lei n. 4.320/64. A dita legislação coloca o MEC na categoria órgão, no sentido de unidade administrativa do governo. A UFSC, por sua vez, se configura como um agrupamento de serviços subordinados ao MEC, ou seja, uma unidade orçamentária. Sua proposta estratégica é estabelecida por meio do Plano de Desenvolvimento Institucional (quinquenal). O PDI é uma exigência, definida no Decreto $n^{\circ} 5.773$, de 9 de maio de 2006, à Universidade para seu recredenciamento junto ao MEC. A elaboração do PDI deve ser pautado no Plano Nacional de Educação, documento este que apresenta o plano decenal para a educação no Brasil.

A conexão entre o PPA e o orçamento anual é feito pela LDO. Aprovada até junho de cada ano para orientar o orçamento do próximo ano, é na Lei de Diretrizes Orçamentárias que serão estabelecidas as prioridades de ações para o exercício seguinte. De acordo com Jund (2006), esta lei, no entanto, não se restringe a definir prioridades, pois possui como obrigação a elaboração de uma série de documentos avaliativos do cumprimento das metas de receitas, despesas, resultado primário, resultado nominal, dívida pública, evolução patrimonial e 
situação atuarial, de modo a consubstanciar as decisões que serão tomadas na etapa seguinte de na previsão de receitas e fixação de despesas do orçamento. Nessa configuração, não se identifica na Universidade documento comparativo ao plano de diretrizes para o próximo exercício. Detentora de autonomia administrativa e financeira, a UFSC, através de seu órgão seccional responsável pelo planejamento e orçamento, tem por responsabilidade a formulação de sua proposta de orçamento anual e encaminhamento ao MEC, independentemente de ter definido prioridades para o exercício seguinte.

\subsection{ANÁliSE DA RELAÇÃO ENTRE A ALOCAÇÃO DE RECURSOS ORÇAMENTÁRIOS E DESEMPENHO DAS AÇÕES DO ORÇAMENTO}

Para proceder com a análise da relação entre o desempenho das ações e os recursos alocados a cada uma delas, foi necessário, inicialmente, calcular a variação $(\Delta)$ dos valores do orçamento e desempenho de cada ação no decorrer do período analisado, conforme apresentado na Tabela 1. A Tabela 1 segue a lógica da do sistema orçamentário adotado no Brasil - o orçamento-programa - no qual o orçamento é composto por programas instrumentos pelos quais as unidades orçamentárias poderão alcançar seus objetivos; e, ações - divisão de programas complexos para facilitar sua execução (GIACOMONI, 2010). Para exemplificar, cita-se o programa 750, desdobrado em ações como a Ação: 0750.2004.26246.0042 - Assistência Médica e Odontológica aos Servidores, Empregados e seus Dependentes. O desempenho da referida ação é mensurado por meio do indicador número de pessoas atendidas.

Tabela 1 Variação do desempenho e do orçamento no período

\begin{tabular}{|c|c|c|c|c|c|c|c|c|c|c|c|}
\hline \multirow{2}{*}{ Programa } & \multirow{2}{*}{ Ação } & \multicolumn{2}{|c|}{2007} & \multicolumn{2}{|c|}{2008} & \multicolumn{2}{|c|}{2009} & \multicolumn{2}{|c|}{2010} & \multicolumn{2}{|c|}{2011} \\
\hline & & D & $\mathbf{O}$ & D & $\mathbf{O}$ & D & $\mathbf{O}$ & D & $\mathbf{O}$ & D & $\mathbf{O}$ \\
\hline 89 & 181 & 0,00000 & 0,00000 & 0,04448 & 0,01142 & $-0,05483$ & 0,16548 & $-0,1928$ & 0,3864 & $-0,9998$ & 0,5228 \\
\hline 89 & 181 & 0,00000 & 0,00000 & 0,00000 & 0,00000 & 0,00000 & 0,00000 & 0,0000 & 0,0000 & $\begin{array}{ll}-0,9981 \\
\end{array}$ & 8,5685 \\
\hline 167 & 4013 & 0,00000 & 0,00000 & 0,00000 & 0,00000 & 0,00000 & 0,00000 & 0,0000 & $-1,0000$ & 0,0000 & $-1,0000$ \\
\hline 681 & 329 & 0,00000 & 0,00000 & 0,07943 & $-0,18869$ & 0,00000 & $-1,00000$ & 0,0000 & $-1,0000$ & 0,0000 & $-1,0000$ \\
\hline 681 & 332 & 0,00000 & 0,00000 & 0,00000 & $-0,19137$ & 0,00000 & $-1,00000$ &, 0000 & $-1,0000$ & 0,0000 & $-1,0000$ \\
\hline 681 & 333 & 0,00000 & 0,00000 & 0,00000 & $-0,52000$ & 0,00000 & $-1,00000$ & 0,0000 & $-1,0000$ & 0,0000 & $-1,0000$ \\
\hline 681 & 334 & 0,00000 & 0,00000 & 0,00000 & $-0,13999$ & 0,00000 & $-1,00000$ & 0,0000 & $-1,0000$ & 0,0000 & $-1,0000$ \\
\hline 681 & 342 & 0,00000 & 0,00000 & 0,00000 & $-0,19242$ & 0,00000 & $-1,00000$ & 0,0000 & $-1,0000$ & 0,0000 & $-1,0000$ \\
\hline 681 & 321 & 0,00000 & 0,00000 & 0,02270 & $-0,11085$ & 0,00000 & $-1,00000$ & 0,0000 & $-1,0000$ & 0,0000 & $-1,0000$ \\
\hline 681 & 325 & 0,00000 & 0,00000 & 0,00000 & $-0,19169$ & 0,00000 & $-1,00000$ & 0,0000 & $-1,0000$ & 0,0000 & $-1,0000$ \\
\hline 681 & 328 & 0,00000 & 0,00000 & $\begin{array}{l}-0,01593 \\
\end{array}$ & $-0,05355$ & 0,00000 & $-1,00000$ & 0,0000 & $-1,0000$ & 0,0000 & $-1,0000$ \\
\hline 750 & 2004 & 0,00000 & 0,00000 & 0,00000 & 0,88202 & $-0,76663$ & 7,28616 & $-0,4584$ & 3,8196 & $-0,6645$ & 6,4708 \\
\hline 750 & 2010 & 0,00000 & 0,00000 & $\begin{array}{l}-0,00888 \\
\end{array}$ & 0,08818 & 0,38661 & $-0,18589$ & 0,0046 & $\begin{array}{l}-0,1301 \\
\end{array}$ & $-0,0643$ & $-0,5213$ \\
\hline 750 & 2011 & 0,00000 & 0,00000 & 0,01726 & 0,32271 & 0,30750 & $-0,37194$ & 0,0144 & $-0,4516$ & $-0,1124$ & $-0,6101$ \\
\hline 750 & 2012 & 00000 & 0,00000 & 0,01417 & 0,01457 & 0,27880 & $-0,18616$ & $-0,5845$ & 1,0677 & $-0,5709$ & 0,9697 \\
\hline 750 & $20 \mathrm{CW}$ & 0,00000 & 0,00000 & 0,00000 & 0,00000 & 0,00000 & 0,00000 & 0,0000 & 0,0000 & 0,0000 & $-0,4917$ \\
\hline
\end{tabular}




\section{ORÇAMENTO POR DESEMPENHO: UMA ANÁLISE DA RELAÇÃO ENTRE DESEMPENHO E ALOCAÇÃO DE RECURSOS EM AÇÕES NO ORÇAMENTO DE UMA INSTITUIÇÃO FEDERAL DE ENSINO SUPERIOR \\ DOI: http://dx.doi.org/10.5007/1983-4535.2014v7n1p148}

\begin{tabular}{|c|c|c|c|c|c|c|c|c|c|c|c|}
\hline 750 & 2004 & 0,00000 & 0,00000 & 0,00000 & 0,00000 & 0,00000 & 0,00000 & 0,0000 & 0,0000 & 0,0000 & 0,3153 \\
\hline 750 & 2010 & 0,00000 & 0,00000 & 0,00000 & 0,00000 & 0,00000 & 0,00000 & 0,0000 & 0,0000 & $-0,0609$ & 0,1498 \\
\hline 750 & 2011 & 0,00000 & 0,00000 & 0,00000 & 0,00000 & 0,00000 & 0,00000 & 0,0000 & 0,0000 & $-0,1417$ & 0,0635 \\
\hline 750 & 2012 & 0,00000 & 0,00000 & 0,00000 & 0,00000 & 0,00000 & 0,00000 & 0,0000 & 0,0000 & $\begin{array}{l}-0,1318 \\
\end{array}$ & 0,0953 \\
\hline 750 & $20 \mathrm{CW}$ & 0,00000 & 0,00000 & 0,00000 & 0,00000 & 0,00000 & 0,00000 & 0,0000 & 0,0000 & 0,0000 & 0,0000 \\
\hline 750 & 2003 & 0,00000 & 0,00000 & 0,00000 & 0,00000 & 0,00000 & 0,00000 & 0,0000 & $-1,0000$ & 0,0000 & $-1,0000$ \\
\hline 901 & 5 & 0,00000 & 0,00000 & \begin{tabular}{|l|}
$-0,87231$ \\
\end{tabular} & 6,83135 & $-0,63899$ & 1,77003 & $-0,7907$ & 3,8091 & 0,4108 & $-0,2912$ \\
\hline 901 & 00G5 & 0,00000 & 0,00000 & 0,00000 & 0,00000 & 0,00000 & 0,00000 & 0,0000 & 0,0000 & 0,0000 & $-0,8645$ \\
\hline 1061 & 2991 & 0,00000 & 0,00000 & 0,27853 & 0,13294 & 0,05232 & 0,36858 & $-0,0776$ & 0,6943 & $-0,1960$ & 1,3270 \\
\hline 1061 & 8429 & 0,00000 & 0,00000 & 0,00000 & 0,00000 & 0,00000 & 0,00000 & 0,0000 & 0,0000 & 1,2214 & $-0,5209$ \\
\hline 1062 & 2992 & 0,00000 & 0,00000 & 0,12593 & 0,29043 & $-0,18476$ & 0,90441 & 0,0000 & $-1,0000$ & 0,0000 & $-1,0000$ \\
\hline 1067 & 4572 & 0,00000 & 0,00000 & 0,29674 & 0,00000 & 0,90191 & 0,03753 & 0,2576 & 0,9190 & $-0,3410$ & 1,3276 \\
\hline 1067 & 4572 & 0,00000 & 0,00000 & 0,00000 & 0,00000 & 0,00000 & 0,00000 & 0,0000 & 0,0000 & 0,0000 & 0,0000 \\
\hline 1073 & 4002 & 0,00000 & 0,00000 & 0,82859 & 0,41587 & $-0,57001$ & 4,19843 & $-0,7453$ & 4,3103 & $-0,6406$ & 5,2592 \\
\hline 1073 & 4004 & 0,00000 & 0,00000 & 0,08381 & 0,60928 & 0,03296 & 2,01571 & $-0,1395$ & 2,0335 & $-0,0112$ & 4,0110 \\
\hline 1073 & 4008 & 0,00000 & 0,00000 & 2524,68998 & 0,10267 & 6440,16209 & 0,57524 & 1620,9859 & 1,6254 & 9933,0964 & 1,6254 \\
\hline 1073 & 4009 & 0,00000 & 0,00000 & $-0,10199$ & 0,15682 & $-0,04966$ & 0,14833 & $-1,0000$ & 0,3573 & $-0,0878$ & 0,3977 \\
\hline 1073 & $09 \mathrm{HB}$ & 0,00000 & 0,00000 & 0,00000 & 0,13124 & $-0,02486$ & 0,10686 & $\begin{array}{l}-0,1379 \\
\end{array}$ & 0,2449 & $-0,1896$ & 0,9373 \\
\hline 1073 & 8282 & 0,00000 & 0,00000 & 0,00000 & 0,00000 & 0,00000 & $\begin{array}{l}0,00000 \\
\end{array}$ & 0,0000 & 0,5986 & $-0,4220$ & 1,8577 \\
\hline 1073 & $11 \mathrm{JK}$ & 0,00000 & 0,00000 & 0,00000 & 0,00000 & 0,00000 & 0,00000 & 0,0000 & 0,5042 & $-0,3524$ & 1,4001 \\
\hline 1073 & 4086 & 0,00000 & 0,00000 & 0,00000 & $-0,99067$ & 0,00000 & $-1,00000$ & 0,0000 & 0,0000 & $-0,0731$ & 0,0682 \\
\hline 1073 & $09 \mathrm{HB}$ & 0,00000 & 0,00000 & 0,00000 & 0,00000 & 0,00000 & 0,00000 & 0,0000 & 0,0000 & $-0,1297$ & 0,1528 \\
\hline 1073 & $125 \mathrm{C}$ & 0,00000 & 0,00000 & 0,00000 & 0,00000 & 0,00000 & 0,00000 & 0,0000 & 0,0000 & 0,0000 & $-1,0000$ \\
\hline 1073 & 2E14 & 0,00000 & 0,00000 & 0,00000 & 0,00000 & 0,00000 & 0,00000 & 0,0000 & $-1,0000$ & 0,0000 & $-1,0000$ \\
\hline 1073 & $7 \mathrm{~J} 15$ & 0,00000 & 0,00000 & 0,00000 & 0,00000 & 0,00000 & 0,00000 & 0,0000 & 0,0000 & 0,0000 & 0,0000 \\
\hline 1073 & $7 \mathrm{~J} 15$ & 0,00000 & 0,00000 & 0,00000 & 0,00000 & 0,00000 & 0,00000 & 0,0000 & 0,0000 & 0,0000 & 0,0000 \\
\hline 1073 & $7 \mathrm{~J} 15$ & 0,00000 & 0,00000 & 0,00000 & 0,00000 & 0,00000 & $\begin{array}{l}0,00000 \\
\end{array}$ & 0,0000 & 0,0000 & 0,0000 & 0,0000 \\
\hline 1073 & $7 \mathrm{~K} 32$ & 0,00000 & 0,00000 & 0,00000 & 0,00000 & 0,00000 & 0,00000 & 0,0000 & 0,0000 & 0,0000 & 0,0000 \\
\hline 1375 & 4006 & 0,00000 & 0,00000 & 0,21563 & 0,00076 & 0,18537 & $\begin{array}{l}0,19789 \\
\end{array}$ & 0,3882 & 0,0637 & 0,2553 & 1,2031 \\
\hline 1375 & 8667 & 0,00000 & 0,00000 & 0,01300 & 0,00000 & $-0,11486$ & 0,33831 & $-0,4343$ & 1,6898 & $-0,0993$ & 1,4950 \\
\hline 1448 & 6333 & 0 & 0 & 0 & 0 & 0 & 0 & 0,0000 & 0,0000 & 0,0000 & $-1,0000$ \\
\hline
\end{tabular}

Fonte: Dados da pesquisa.

A Tabela 1 apresenta a variação do desempenho (D) e a variação do orçamento $(O)$ em cada ação e programa. Com base nessa tabela foi aplicada a estatística descritiva e realizado o cálculo da correlação. Para o teste da hipótese $H 1=\mathrm{O}$ desempenho da ação em determinado ano influência o orçamento do ano posterior, foi possível obter 31 observações válidas, conforme demonstrado na Tabela 2.

Analisando a Tabela 2 é possível evidenciar que a média da variação do desempenho é de 0,1234 enquanto a média da variação do orçamento é de 0,7323 . O máximo da variação de desempenho de um ano para o outro foi de 0,9019 enquanto da variação do orçamento foi de 4,1984, o que mostra uma maior volatilidade na variação do orçamento em relação à variação do desempenho. Após essa etapa, foi calculada a correlação entre a variação do desempenho e a variação do orçamento, como demonstrado na Tabela 3.

Tabela 2 Análise com base em estatística descritiva para os eventos da hipótese H1.

\begin{tabular}{|l|r|r|}
\hline & $\Delta$ Desempenho & \multicolumn{1}{|c|}{$\Delta$ Orçamento } \\
\hline Média & 0,123403332 & 0,732355988 \\
\hline Erro padrão & 0,045840175 & 0,205573262 \\
\hline Desvio padrão & 0,255227291 & 1,144583483 \\
\hline Variância da amostra & 0,06514097 & 1,310071349 \\
\hline
\end{tabular}




\begin{tabular}{|l|r|r|} 
Mínimo & $-0,192823277$ & $-0,610071678$ \\
\hline Máximo & 0,901906965 & 4,198427382 \\
\hline Contagem & 31 & 31 \\
\hline
\end{tabular}

Fonte: Dados da Pesquisa.

Tabela 3 Análise de correlação para os eventos da hipótese H1.

\begin{tabular}{|l|r|}
\hline Correlação & 0,208167362 \\
\hline
\end{tabular}

Fonte: dados da pesquisa.

A Tabela 3 evidência uma correlação entre as variáveis de 0,2081, considerada uma correlação fraca, ou seja, a partir dessa análise a hipótese H1 é refutada. A fraca correlação apresentada demonstra que o desempenho da ação em determinado ano não influência diretamente o orçamento do ano seguinte. Esse resultado vai ao encontro da argumentação dos críticos ao PBB, sobre o papel limitado das informações sobre desempenho na alocação final dos recursos, conforme apresentado por Tat-Kei Ho (2011). O autor argumenta, apesar disso, que, por prover informações sobre o desempenho das organizações públicas, o PBB pode agregar valor ao processo de negociação do orçamento em diferentes fases de sua elaboração.

Para o teste da hipótese $H 2=\mathrm{o}$ aumento do orçamento em relação ao orçamento do ano anterior influência o desempenho da ação no ano seguinte, apresenta-se a Tabela 4, que demonstra a estatística descritiva das observações dessas variáveis.

Tabela 4 Análise com base em estatística descritiva para os eventos da hipótese H2.

\begin{tabular}{|l|r|r|}
\hline & $\Delta$ Orçamento & \multicolumn{1}{|c|}{$\Delta$ Desempenho } \\
\hline Média & 0,353050032 & 0,083477522 \\
\hline Erro padrão & 0,114967229 & 0,041805075 \\
\hline Desvio padrão & 0,796516327 & 0,289634057 \\
\hline Variância da amostra & 0,63443826 & 0,083887887 \\
\hline Mínimo & $-0,610071678$ & $-0,196026721$ \\
\hline Máximo & 4,010977123 & 1,221392972 \\
\hline Contagem & 48 & 48 \\
\hline
\end{tabular}

Fonte: dados da pesquisa.

No teste da hipótese $\mathrm{H} 2$ foi possível obter 48 observações, sendo a média da variação do orçamento de 0,3530 enquanto a variação do desempenho foi de 0,0834 . O máximo da variação do orçamento foi de 4,0109 enquanto o máximo da variação de performance foi de 1,2213. Mais uma vez houve maior volatilidade na variação do orçamento em detrimento da 
variação do desempenho das ações. Após a análise da variação, novamente foi calculada a correlação entre essas variáveis, conforme demonstrado na Tabela 5.

Tabela 5 Análise de correlação para os eventos da hipótese H2.

\begin{tabular}{|l|l|}
\hline Correlação & $-0,234144076$ \\
\hline
\end{tabular}

Fonte: dados da pesquisa.

A Tabela 5 evidência uma correlação entre as variáveis de $-0,234144076$, o que se configura como uma fraca correlação, e acaba por refutar também a hipótese H2. Muitas vezes, na discussão sobre alocação de recursos, emerge a questão sobre qual a melhor decisão a ser tomada no caso de ações que não alcançam seus objetivos. É melhor finalizar a ação ou injetar mais recursos com vistas a melhoria do desempenho da ação? O teste da hipótese $\mathrm{H} 2$ buscou lidar com essa questão. A hipótese considerava que uma vez tendo sido alocados mais recursos para a ação, a tendência seria de melhoria do desempenho no ano seguinte. $\mathrm{O}$ resultado não só refutou a ação, como também, pelo fato de apresentar correlação negativa, pode levar à conclusão que o aumento dos recursos alocados a essas ações leva a uma diminuição de seu desempenho. Ou, no mínimo, o aumento na aplicação de recursos não resultou em melhoria do desempenho para a sociedade.

\section{CONCLUSÕES}

No contexto das discussões desenvolvidas no âmbito do movimento da New Public Management - NPM - sobre a melhoria na alocação de recursos públicos e seguindo uma linha de estudos relacionada ao Performance-based budgenting - PBB - o presente estudo buscou analisar a eficiência da gestão orçamentária da Universidade Federal de Santa Catarina, por meio da análise da relação entre os recursos alocados nas ações do orçamento da instituição e o desempenho dos indicadores de cada uma dessas ações. O estudo foi orientado pela seguinte pergunta de pesquisa, que nesse momento é resgatada e devidamente respondida: Existe correlação positiva entre o desempenho alcançado nos indicadores e os recursos alocados às ações orçamentárias no âmbito da Universidade Federal de Santa Catarina?

Para responder à pergunta de pesquisa proposta foram levantadas duas hipóteses. A hipótese H1 buscou verificar se o aumento do desempenho em determinada ação influenciaria o montante de recursos alocados á ação no período seguinte. A hipótese inicial dos autores, de 
que o desempenho das ações acarretaria aumento do orçamento para a ação no ano seguinte foi refutada devido à fraca correlação encontrada na análise $(0,208167362)$. Essa evidência é corroborada pelos críticos do $\mathrm{PBB}$, que argumentam ser o processo orçamentário um processo de negociação e disputa de poder, sendo que, nesse sentido, as informações sobre desempenho não são consideradas prioritárias no processo de alocação de recursos. Foi evidenciada uma volatilidade superior na variação do orçamento em relação à variação do desempenho das ações, o que demonstra a pouca preocupação com esse tipo de análise no momento da tomada de decisão orçamentária. Apesar dessa constatação, Ho (2011) argumenta que mesmo assim, as informações sobre o desempenho podem apoiar o processo de tomada de decisão orçamentária pelo fato de oferecer aos gestores públicos um referencial de análise no qual possam se basear no momento de negociação pelos recursos.

No que tange à hipótese $\mathrm{H} 2$, esta buscou tratar da necessidade de alocação de recursos em programas com baixo desempenho com vistas ao resgate do mesmo e melhoria de seu desempenho futuro. Dessa forma, a hipótese previa que, nos casos em que foi evidenciado baixo desempenho, maiores montantes seriam alocados com vistas a reverter a situação no período seguinte. Mais uma vez a hipótese foi refutada, dessa vez o resultado da análise apresentou correlação negativa $(-0,234144076)$. Esse resultado pode levar à conclusão de que os recursos públicos são mal alocados, tendo em vista que, apesar dos recursos alocados a essas ações terem aumentado ao longo do tempo, o desempenho da ação tende a cair.

Após o teste das duas hipóteses de pesquisa, pode-se dizer que não existe correlação positiva entre o desempenho alcançado nas ações e os recursos alocados às mesmas no âmbito da organização em análise. Além da negativa em relação à pergunta de pesquisa, a análise dos dados mostrou que em determinados casos os indicadores de desempenho ora não estão definidos, ora não são acompanhados em todo o período de análise. Essa constatação demonstra a necessidade de uma maior discussão sobre os indicadores adotados para avaliação das ações, bem como sobre seu processo de acompanhamento, o que abre espaço para futuras pesquisas.

\section{REFERÊNCIAS}

ARAÚJO, G. M. de. Financiamento do ensino superior: um estudo sobre os recursos do tesouro destinado as IFES e o caso da UNIRIO - 2000 a 2005. 85 f. Dissertação (Mestrado Profissionalizante em Economia e Gestão Empresarial) - Universidade Cândido Mendes, Rio de Janeiro, 2009. 
BOISVERT, H. La comptabilité de management: price de décision et gestion. 2. ed. Québec: ERPI Éditions Du Renouveau, 1999.

BRASIL. Presidência da República. Decreto no 5.773, de 9 de maio de 2006. Dispõe sobre o exercício das funções de regulação, supervisão e avaliação de instituições de educação superior e cursos superiores de graduação e seqüenciais no sistema federal de ensino.

BROTTI, V.; LAFFIN, M.; BORGERT, A. Orçamento público: levantamento dos artigos, dissertações e teses no Brasil entre 1980 e 2005 em algumas das principais fontes de publicações disponíveis em meio eletrônico. In.: Congresso USP de Controladoria e Contabilidade...Anais...São Paulo, 26 e 27 de Julho de 2007.

BRUMBY, J.; ROBINSON, M. Does performance budget works? an analytical review of the empirical literature. . Washington DC: [s.n.]. , 2005

BRYMAN, A. The debate about quantitative and qualitative research. In: BRYMAN, A. (Ed.). Quantity and quality in social research. London: Unwin Hyman, 1988a. .

BRYMAN, A. The nature of quantitative research. In: BRYMAN, A. (Ed.). Quantity and quality in social research. London: Unwin Hyman, 1988b.

CALIL, V. L. L. O. Uma contribuição ao aperfeiçoamento da elaboração das propostas orçamentárias da Universidade Estadual do Oeste do Paraná - UNIOESTE. $117 \mathrm{f}$. Dissertação (Mestrado em Administração) - Universidade Federal de Santa Catarina, Florianópolis, 2000.

CAVALCANTE, P. L. Orçamento por desempenho: uma analise qualitativa comparada dos modelos de avaliação dos programas governamentais no Brasil e nos Estados Unidos. Revista de Gestão USP, v. 17, n. 1, p. 13-25, 2010.

FERREIRA, J. O. L. Orçamento participativo: uma proposta para a UFPE - Universidade Federal de Pernambuco. 141 f. Dissertação (Mestrado Profissionalizante em Gestão Pública para o Desenvolvimento do Nordeste) - Universidade Federal de Pernambuco, Recife, 2003.

FRANCISCO, J. G. A integração entre o planejamento e o orçamento público nas Instituições de Federais de Ensino Superior: um estudo de caso múltiplo. 109 f. Dissertação (Mestrado Profissionalizante em Sistemas de Gestão) - Universidade Federal Fluminense, Rio de Janeiro, 2005.

GIACOMONI, J. Orçamento público. 15. ed. São Paulo: Atlas, 2010.

GIL, A. C. Como elaborar projetos de pesquisa. 4. ed. São Paulo: Atlas, 2007.

GRIZZLE, G. A.; PETTIJOHN, C. D. Implementing Performance-Based Program Budgeting: a system-dynamics perspective. Public Administration Review, v. 62, n. 1, p. 51-62, 2002.

HO, A. T. PBB in American Local Governments: it's more than a management tool. Public Administration Review, v. 71, n. 3, p. 391-401, 2011. 
JUND, S. Administração, orçamento e contabilidade pública. 1. ed. Rio de Janeiro, Elsevier, 2006.

KONG, D. Performance-based Budgeting: the U.S. experience. Public Organization Review, v. 5, n. 2, p. 91-107, 2005.

LARKLEY, P. D.; DEVEREUX, E. A. Good budgetary decision processes. In: FREDERICKSON, H. G.; JONHSTON, J. M. (Eds.). Public management reform and Innovation: research, theory and applications. Tuscaloosa: University of Alabama Press, 1999. p. 168-188.

LEITE, R. M.; CHEROBIM, A. P. M. S.; SILVA, H. DE F. N.; BUFREM, L. S. Orçamento empresarial: levantamento da produção científica no período de 1995 a 2006 . Revista Contabilidade e Finanças, v. 19, n. 47, p. 56-72, ago 2008.

LIBBY, T.; LINDSAY, R. M. Beyond budgeting or budgeting reconsidered? a survey of North-American budgeting practice. Management accounting review, v. 2, n. 1, p. 56-75, 2010 .

LUNKES, R. J. Manual de orçamento. 2. ed. São Paulo: Atlas, 2009. p. 176

OSTERGREN, K.; STENSAKER, I. Management control without budgets: a field study of "beyond budgenting" in practice. European accouting review, v. 20, n. 1, p. 149-181, 2011.

PAIVA, S. A. R. Políticas de financiamento para a educação superior: avaliação institucional e gestão orçamentária na Universidade Federal de Uberlândia/UFU (1995-2008). 124 f. Dissertação (Mestrado) - Universidade Federal de Uberlândia, Uberlândia, 2009.

PIRES, J. S. D. B. A plenitude da autonomia financeira e o ciclo orçamentário das Universidades Públicas do Estado do Paraná. 231 f. Dissertação (Mestrado em Engenharia da Produção) - Universidade Federal de Santa Catarina, Florianópolis, 2001.

RADIN, B. A. Challenging the performance movement: accountability, complexity, and democratic values. Washington DC: Georgetown University Press, 2006.

ROBINSON, MARC. Best practice in performance budgeting. Annual Conference of the Association for Budgeting and Financial Management. Anais... Kansas City: [s.n.]. , 2002

ROSA, F. S. DA; ENSSLIN, S. R.; ENSSLIN, L.; LUNKES, R. J. Gestão da evidenciação ambiental: um estudo sobre as potencialidades e oportunidades do tema. Engenharia Sanitária Ambiental, v. 16, n. 1, p. 157-166, 2011.

RICHARDSON, R. J. Pesquisa Social: métodos e técnicas. 3. ed. São Paulo: Atlas, 1999.

RUBIN, I. S. The politics of public budgeting: getting and spending, borrowing and balancing. Washington DC: CQ Press, 2010.

SECCHI, L. Modelos organizacionais e reformas na administração pública. Revista de Administração Pública, Rio de Janeiro, v. 43, n. 2, p. 347-369, Mar./abr., 2009. 
UYAR, A. An evaluation of budgeting approaches: traditional budgenting, better budgeting and beyond budgeting. Journal of academic studies, v. 11, n. 42, p. 113-130, 2009.

VASCONCELlOS, G. M. A Gestão Orçamentária da UFS no período de 1998 a 2008. 172 f. Dissertação (Mestrado Profissional em Desenvolvimento Regional) - Universidade Federal de Sergipe, São Cristóvão, 2010.

WAGGONER, D. B.; NEELY, A.; KENNERLEY, M. The forces that shape organizational performance measurement systems: an interdisciplinary review. International Journal of Production Economics, v. 60-61, n. 1, p. 53-60, Abr., 1999.

ZDANOWICZ, J. E. Orçamento operacional. Porto Alegre: Sagra, 1983. 